\title{
SHOC2 is associated with the survival of breast cancer cells and has prognostic value for patients with breast cancer
}

\author{
WENWEN GENG ${ }^{1,2}, \mathrm{KE} \mathrm{DONG}^{1}$, QIAN PU ${ }^{2}$, \\ YANRONG $\mathrm{LV}^{1}$ and HAIDONG GAO ${ }^{1,2}$ \\ ${ }^{1}$ Department of Breast Surgery, Qilu Hospital of Shandong University, Jinan, Shandong 250012; \\ ${ }^{2}$ Department of Breast Surgery, Qilu Hospital (Qingdao) of Shandong University, Qingdao, Shandong 266000, P.R. China
}

Received June 21, 2019; Accepted November 27, 2019

DOI: $10.3892 / \mathrm{mmr} .2019 .10889$

\begin{abstract}
SHOC2 leucine rich repeat scaffold protein (SHOC2) has been identified as a positive regulator of the Ras pathway; however, the function of SHOC2 in breast cancer has rarely been explored. The current study investigated the effects of SHOC2 on breast cancer cell growth and evaluated its prognostic value in patients with breast cancer. The effects of SHOC2 on MCF-7 and MDA-MB-231 breast cancer cells were studied using short hairpin RNA. In total, 120 pairs of formalin-fixed, paraffin-embedded breast cancer tissue specimens were compared to normal tissue using immunohistochemical staining. SHOC2 knockdown significantly inhibited MCF-7 and MDA-MB-231 breast cancer cell proliferation, and induced cell apoptosis and cell cycle arrest. Additionally, the RAS-MAPK/PI3K pathway was inhibited by SHOC2 knockdown. In a clinical study, the results revealed that high SHOC2 expression was associated with more aggressive clinical characteristics of breast cancer. Moreover, Kaplan-Meier and Cox regression analyses indicated that SHOC2 expression was an independent prognostic factor for survival, suggesting that increased SHOC2 expression predicted a worse overall survival. This indicated that SHOC2 knockdown could affect breast cancer cell survival, and SHOC2 upregulation may be associated with a poor prognosis in patients with breast cancer.
\end{abstract}

\section{Introduction}

Breast cancer is the most common type of cancer in women worldwide, and it is estimated that 1.4 million women a year receive a diagnosis of breast cancer, while 458,000 succumb to the disease (1). Breast cancer involves a multi-step process

Correspondence to: Dr Haidong Gao, Department of Breast Surgery, Qilu Hospital of Shandong University, 107 Wenhua West Road, Lixia, Jinan, Shandong 250012, P.R. China

E-mail: haidonggao@sdu.edu.cn

Key words: SHOC2 leucine rich repeat scaffold protein, breast cancer, proliferation, apoptosis, survival associated with the abnormal expression of several oncogenes and tumor suppressor genes (2). Numerous types of malignant tumors are associated with the abnormal expression of Ras family genes (3). A more comprehensive understanding of the Ras oncogene pathways could lead to improved strategies for therapy and prevention.

SHOC2 leucine rich repeat scaffold protein (SHOC2) was first isolated in Caenorhabditis elegans (4) and was identified as a positive regulator of the Ras pathway (5). SHOC2 is a scaffold protein that binds components of the Ras pathway and modulates their functions $(6,7)$. Ras binds directly to SHOC2 to form a complex that binds to the catalytic subunit of protein phosphatase 1 (PP1c); this Ras/SHOC2/PP1c complex activates the Ras pathway by dephosphorylating Raf-1 proto-oncogene, serine/threonine kinase (Raf-1) at S259 (8). SHOC2 has been reported to regulate the Ras signaling cascade in a number of ways; in particular, it has essential roles in embryogenesis and normal biological processes $(9,10)$. Furthermore, the role of SHOC2 in various types of malignant cells has been examined, which has highlighted its potential role in tumorigenesis (11). Lee et al (12) demonstrated that SHOC2 promoted colorectal tumorigenesis and metastasis via ERK and PI3K-AKT signaling pathways. Kaduwal et al (13) suggested that SHOC2 also served an essential role in the process of metastasis via the Ras-PI3K-Rac-matrix metalloproteinase signaling pathway in human melanoma. In addition, Kaplan et al (14) demonstrated that SHOC2 was associated with the mechanisms of acquired resistance to the Raf inhibitor vemurafenib. In this previous study, SHOC2 could alter signaling connections and re-route oncogenic Ras signals to Raf-1 in order to mediate reactivation of the ERK1/2 pathway and facilitate drug resistance in cells (14). SHOC2 has also been shown to be a positive regulator that contributes to the malignant properties of different tumor cells by modulating the growth, transformation, migration and invasion of cancer cells $(10,15,16)$. Our preliminary experiments revealed that SHOC2 was highly expressed in MCF-7 and MDA-MB-231 breast cancer cells (data not shown). However, the function of SHOC2 in breast cancer has rarely been explored. In this study, the association between SHOC2 upregulation and the clinicopathological features of breast cancer was investigated, and the prognostic value of SHOC2 was evaluated. Furthermore, the effects of 
SHOC2 on growth, apoptosis and cell cycle progression in breast cancer were elucidated.

\section{Materials and methods}

Patients and tissues. A total of 120 patients with breast cancer who underwent surgical treatment and had complete tumor tissue blocks preserved at the Department of Breast Surgery, Qilu Hospital of Shandong University (Jinan, China) between January 2004 and May 2016 were recruited. All 120 patients were female, and their ages ranged between 26 and 72 years old, with an average age of $46.3 \pm 9.9$ years old. The inclusion criteria were as follows: Diagnosed with breast cancer based on the post-operative pathological section; completed clinical follow-up survey; $>18$ years old; and never received pre-operative chemotherapy or radiotherapy. The exclusion criteria were as follows: $<18$ years old; had incomplete clinical follow-up data or other malignant tumors at the same time; or received pre-operative chemotherapy or radiotherapy. Patients all underwent surgery: 88 had modified radical mastectomy $(73 \%)$ and 32 had breast-conserving therapy (27\%). After surgery, the patients were treated with chemotherapy or radiotherapy. Indications for post-operative chemotherapy, radiotherapy or chemoradiotherapy were patients with various pathological features, such as advanced cancer stage, lymph node positivity, perineural invasion, lymphovascular invasion and receptor status. Patients positive for hormone receptors received adjuvant endocrine therapy for 5 years and patients positive for human epidermal growth factor receptor 2 (HER2) received trastuzumab therapy for 1 year. After finishing treatment, patients were regularly followed-up with clinical examinations and imaging. Patients were scheduled for clinical visits every 4-6 months during the first and second years, every 6 months during the third, fourth and fifth years, and annually thereafter.

Immunohistochemistry (IHC). Formalin-fixed (10\% neutral buffered formalin at $4^{\circ} \mathrm{C}$ for $12-24 \mathrm{~h}$ at room temperature), paraffin-embedded sections (size, $4 \mu \mathrm{m}$ ) from breast cancer and normal breast tissue samples were obtained. The normal tissue samples were obtained from the adjacent tissue of the same patients, and the distance between tumor and normal tissues was $\geq 0.5 \mathrm{~cm}$ (17). The sections were deparaffinized, rehydrated in a descending alcohol series and heated at $120^{\circ} \mathrm{C}$ for $10 \mathrm{~min}$ in $10 \mathrm{mM}$ sodium citrate $(\mathrm{pH} \mathrm{6.0)}$ to retrieve antigens. Endogenous peroxidase activity was quenched with $3 \%$ hydrogen peroxide at room temperature for $10 \mathrm{~min}$. The sections were then incubated with an anti-SHOC2 antibody (1:400; cat. no. Fnab06912; Wuhan Fine Biotech Co., Ltd.) at room temperature for $1 \mathrm{~h}$, followed by a horseradish peroxidase (HRP)-conjugated secondary antibody (cat. no. 8114; Cell Signaling Technology, Inc.) at room temperature for $15 \mathrm{~min}$. The sections were developed in diaminobenzidine for $5 \mathrm{~min}$ and counterstained with hematoxylin for $3 \mathrm{~min}$ at room temperature. The primary antibody was omitted for the negative controls. Sections were visualized and photographed using an optical microscope (Leica Microsystems $\mathrm{GmbH}$; magnification, $\mathrm{x} 50$ and $\mathrm{x} 200$ ). The expression levels of SHOC2 were semi-quantified using a semi-quantitative
IHC scoring system, as previously described $(18,19)$. The percentage of positive tumor cells was graded on a scale between 0 and 4 , as follows: 0 , none; $1,1-10 \% ; 2,11-50 \%$; 3 , $51-80 \% ; 4,>80 \%$. The intensity of staining was graded on a scale between 0 and 3, as follows: 0 , none; 1 , weak staining; 2 , moderate staining; 3 , strong staining. The combination of the extent (E) and intensity (I) of staining was obtained as the product of $\mathrm{E}$ and $\mathrm{I}(\mathrm{EI})$, which varied between 0 and 12 for each sample. Using the X-tile software program (version 3.6.1; The Rimm Lab, Yale University; https://medicine. yale.edu/lab/rimm/research/software.aspx), a significant cutoff point for SHOC2 was identified in terms of overall survival (OS) in patients with breast cancer, as previously described (20). A cutoff score of 8 was selected; 0-8 was considered low expression, whereas $>8$ was considered high expression.

Cell culture and infection. MCF-7 and MDA-MB-231 breast cancer cell lines and the 293T cell line were purchased from The Cell Bank of Type Culture Collection of the Chinese Academy of Sciences and maintained by the Center Lab of Shandong University. Cells were cultured in DMEM (Gibco; Thermo Fisher Scientific, Inc.) supplemented with $10 \%$ FBS (Gibco; Thermo Fisher Scientific, Inc.) and $100 \mathrm{U} / \mathrm{ml}$ penicillin-streptomycin, and grown at $37^{\circ} \mathrm{C}$ in $5 \% \mathrm{CO}_{2}$. Stable knockdown of SHOC2 in the breast cancer cell line was performed using lentiviruses. Briefly, the target sequence (5'-TGCTTGATTTACGGCATAA-3') for short hairpin RNA (shRNA)-SHOC2 and the nonspecific control sequence (5'-TTCTCCGAACGTGTCACGT-3') (21) were inserted into the GV493 shRNA vector (Shanghai GeneChem Co., Ltd.) linearized with AgeI and EcoRI. For GFP expression in this vector, GFP-IRES was cloned in front of the puromycin-resistant marker gene. Virus production was carried out using 293T cells. Briefly, cells were transfected with lentiviral DNA constructs alongside the lentiviral packaging plasmids pHelper 1.0 and pHelper 2.0 (Shanghai GeneChem Co., Ltd.) at a ratio of 4:3:2. The viral supernatants were harvested $48 \mathrm{~h}$ post-transfection, filtered using a $0.45-\mu \mathrm{m}$ pore filter, and used for infection. To establish stable cell lines, cells were seeded in 6 -well plates $\left(2 \times 10^{5} /\right.$ well $)$ until they reached $60-70 \%$ confluence before being infected with either SHOC2 knockdown or the control lentivirus (MOI=10), and selected with puromycin $(2 \mu \mathrm{g} / \mathrm{ml})$ to obtain single-cell clones. The single-cell clones obtained were cultured for 2 weeks, and amplified for further experiments in culture media containing puromycin $(1 \mu \mathrm{g} / \mathrm{ml})$.

Reverse transcription-quantitative (RT-q)PCR. RT-qPCR was performed to detect the mRNA expression levels of SHOC2 in 11 pairs of fresh breast cancer and adjacent noncancerous human breast tissues. Total RNA was isolated from breast cancer and adjacent noncancerous tissues using TRIzol ${ }^{\circledR}$ reagent (Invitrogen; Thermo Fisher Scientific, Inc.). Random-primed cDNA synthesis was performed using a QuantiTect ${ }^{\circledR}$ RT kit (Qiagen $\mathrm{GmbH}$ ). The RT reaction was performed as follows: Initial incubation at $42^{\circ} \mathrm{C}$ for $2 \mathrm{~min}$, followed by sequential steps at $42^{\circ} \mathrm{C}$ for $15 \mathrm{~min}$, and at $95^{\circ} \mathrm{C}$ for $3 \mathrm{~min}$. RT-qPCR was performed using a PTC- $100^{\circledR}$ Thermal cycler (MJ Research, Inc.) with a PrimeScript ${ }^{\mathrm{TM}} \mathrm{RT}$ 
Reagent kit (Takara Biotechnology Co., Ltd.), according to the manufacturer's protocols. For qPCR, the thermocycling conditions were: Pre-denaturation at $95^{\circ} \mathrm{C}$ for $30 \mathrm{sec}$, followed by 40 cycles of denaturation at $95^{\circ} \mathrm{C}$ for $5 \mathrm{sec}$ and of annealing at $60^{\circ} \mathrm{C}$ for $30-60 \mathrm{sec}$. For amplification, the following primers were designed: SHOC2, forward 5'-TCAGTGGTGTATAGG CTGGATTCT-3', reverse 5'-GCTACATCCAGCGTAATG AGGT-3'; GAPDH, forward 5'-CCTCCGGGAAACTGTGGC GTGATGG-3' and reverse 5'-AGACGGCAGGTCAGGTCC ACCACTG-3'. Each sample was examined three times, and the levels of PCR product were adjusted to GAPDH, which served as an internal control. The fold change of mRNA expression was calculated using the formula: $2^{-\Delta \Delta \mathrm{Cq}}(22)$.

Western blot analysis. Confluent cells were washed twice with ice-cold PBS and lysed in RIPA buffer with $1 \mathrm{mM}$ PMSF (Beyotime Institute of Biotechnology). The concentrations of the total protein extracts were determined by the bicinchoninic acid (BCA) method using a commercial kit from Pierce; Thermo Fisher Scientific, Inc. Equal amounts of protein samples $(40 \mu \mathrm{g})$ were separated by SDS-PAGE on $10 \%$ gels and transferred onto PVDF membranes. After blocking with $5 \%$ non-fat milk at room temperature for $1 \mathrm{~h}$, the membranes were incubated overnight at $4^{\circ} \mathrm{C}$ with primary antibodies against SHOC2 (1:1,000; cat. no. Fnab06912; Wuhan Fine Biotech Co., Ltd.), Bcl-2 (1:1,000; cat. no. 15071; Cell Signaling Technology, Inc.), Bax (1:1,000; cat. no. 5023; Cell Signaling Technology, Inc.), poly (ADP-ribose) polymerase (PARP) (1:1,000; cat. no. 9532; Cell Signaling Technology, Inc.), phosphorylated (p)-AKT (1:1,000; cat. no.4060; Cell Signaling Technology, Inc.), AKT (1:1,000; cat. no. 4691; Cell Signaling Technology, Inc.), p-ERK (1:1,000; cat. no. 4376; Cell Signaling Technology, Inc.), ERK (1:1,000; cat. no. 4696; Cell Signaling Technology, Inc.) and GAPDH (1:1,000; cat. no. AP7873a; Abgent, Inc.), followed by incubation with either HRP-conjugated Anti-Rabbit secondary antibodies (1:5,000; cat. no. R4880; Sigma-Aldrich; Merck KGaA) or Anti-Mouse secondary antibodies (1:5,000; cat. no. 7077; Cell Signaling Technology, Inc.) at room temperature for $1 \mathrm{~h}$. Protein bands were detected by ECL western blot substrate (Thermo Fisher Scientific, Inc.). Immunoreactive bands were visualized by ImageQuant LAS 4000 series (GE Healthcare). Image J v.1.43 (National Institutes of Health) software was used to quantify relative protein expression.

MTT assay. Cells ( $5 \times 10^{3} /$ well) were seeded and incubated in 96-well plates at $37^{\circ} \mathrm{C}$ in a $5 \% \mathrm{CO}_{2}$ environment for $1,2,3$, 4 and 5 days. At each time point, $5 \mathrm{mg} / \mathrm{ml} \mathrm{MTT}$ was added to each well. After incubation at $37^{\circ} \mathrm{C}$ for $4 \mathrm{~h}$, the supernatants were removed. Subsequently, $100 \mu \mathrm{l}$ DMSO was added to each well, and the well contents were thoroughly mixed for $5 \mathrm{~min}$. The absorbance was measured at $490 \mathrm{~nm}$ using a microplate reader (TECAN Infinite ${ }^{\circledR} 200$; Tecan Group, Ltd.).

Cell cycle and apoptosis analysis. Cells were cultured to $85 \%$ confluence, collected and then rinsed with cold D-Hanks balanced salt solution. After centrifugation at 1,000 x $\mathrm{g}$ for 5 mins at room temperature, the supernatants were removed, and the cells were fixed in $75 \%$ cold ethanol for $1 \mathrm{~h}$. The fixed cells were rinsed with D-Hanks solution and permeabilized with $0.1 \%$ Triton X-100 and $2 \mathrm{mg} / \mathrm{ml}$ RNase A in D-Hanks solution for $30 \mathrm{~min}$ at $37^{\circ} \mathrm{C}$. The cells were then rinsed with D-Hanks solution and stained with $50 \mathrm{mg} / \mathrm{ml}$ propidium iodide (Sigma-Aldrich; Merck KGaA) at $4^{\circ} \mathrm{C}$ for $1 \mathrm{~h}$. The stained cells were analyzed with a Millipore Guava ${ }^{\circledR}$ easyCyte 5HT flow cytometer (EMD Millipore). A total of 8 days after lentiviral infection, cells $\left(5 \times 10^{5}\right)$ were collected and rinsed with cold D-Hanks balanced salt solution. After centrifugation at $1,000 \mathrm{xg}$ for $3 \mathrm{mins}$ at room temperature, the supernatants were removed. The cells were resuspended with $200 \mu \mathrm{l}$ binding buffer and then stained with $10 \mu \mathrm{l}$ Annexin V-APC (cat. no. 88-8007; eBioscience; Thermo Fisher Scientific, Inc.) at room temperature in the dark for 10-15 min. Subsequently, flow cytometry was performed using the Guava easyCyte 5HT flow cytometer. Data were analyzed using FlowJo software v.10 (FlowJo LLC).

Statistical analysis. All results were repeated three times and the data are presented as the mean \pm standard deviation. The association among SHOC2 expression, proliferation and apoptosis was assessed using unpaired Student's t-test. The mRNA expression was compared between normal and tumor tissues using paired Student's t-test. IHC data were compared between normal and tumor tissues using Wilcoxon signed-rank test. The relationship between SHOC2 expression and clinicopathological characteristics was analyzed by the $\chi^{2}$ test. Survival curves were plotted by the Kaplan-Meier method and compared using the log-rank test. Survival data were evaluated using univariate and multivariate Cox regression analyses. All statistical analyses were performed using SPSS version 18.0 (SPSS, Inc.). $\mathrm{P}<0.05$ was considered to be statistically significant.

\section{Results}

SHOC2 knockdown impairs breast cancer proliferation, and induces apoptosis and cell cycle arrest. MCF-7 and MDA-MB-231 breast cancer cells were infected with shRNA-SHOC2 to induce SHOC2 knockdown (Fig. 1A). Subsequently, MTT assays were performed to determine the effects of SHOC2 on breast cancer cell proliferation, which revealed that cell counts were significantly decreased by SHOC2 knockdown. Therefore, SHOC2 knockdown may inhibit the growth of MCF-7 and MDA-MB-231 breast cancer cells $(\mathrm{P}<0.001$; Fig. $1 \mathrm{~B}$ and $\mathrm{C})$. The mechanism underlying the antiproliferative effects of SHOC2 knockdown was examined by analyzing apoptosis using Annexin V staining. The number of Annexin V-positive cells was significantly higher in shRNA-SHOC2 breast cancer cells than in control cells $(\mathrm{P}<0.001$; Fig. $1 \mathrm{~F}$ and $\mathrm{G})$. Moreover, cell cycle distribution was analyzed using flow cytometry, which showed that SHOC2 knockdown led to cell arrest in the G1 and G2/M phases $(\mathrm{P}<0.05$; Fig. 1D and $\mathrm{E})$. Next, a series of cellular apoptosis-related proteins were analyzed; the expression levels of the anti-apoptosis marker Bcl-2 were significantly decreased, whereas the levels of the pro-apoptotic markers Bax and cleaved-PARP were increased in shRNA-SHOC2 cells $(\mathrm{P}<0.001$; Fig. 2A). These results indicated that knockdown of SHOC2 significantly inhibited proliferation, and promoted apoptosis and cell cycle arrest in breast cancer cells. 
A

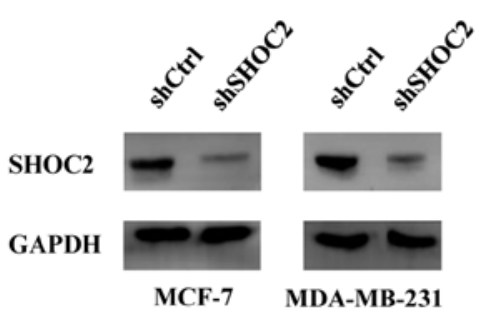

D

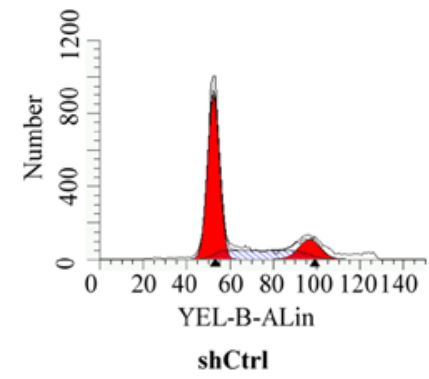

$\mathbf{E}$

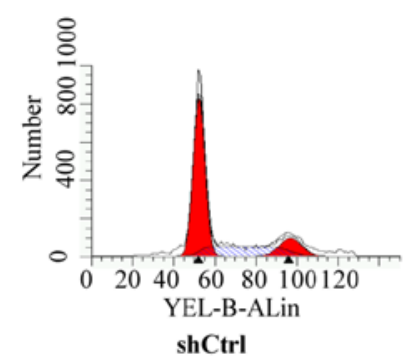

$\mathbf{F}$

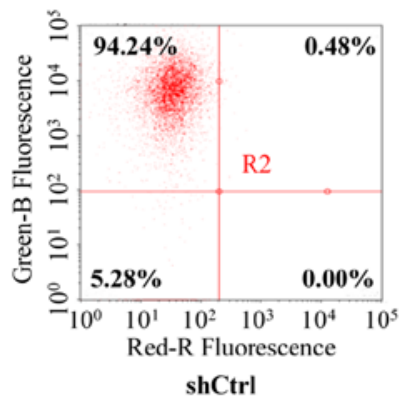

G

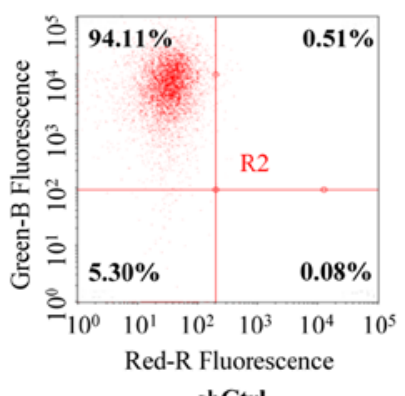

B
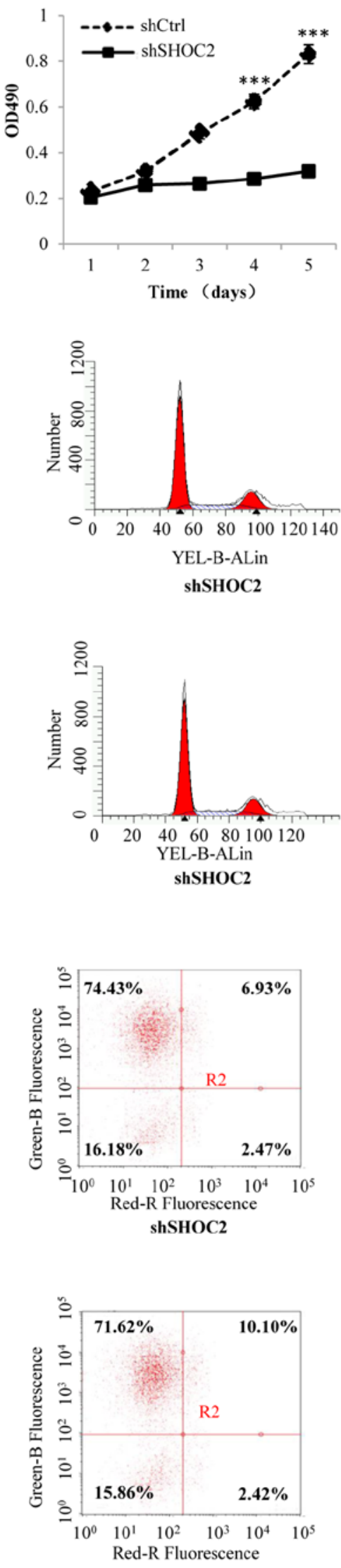

shSHOC2
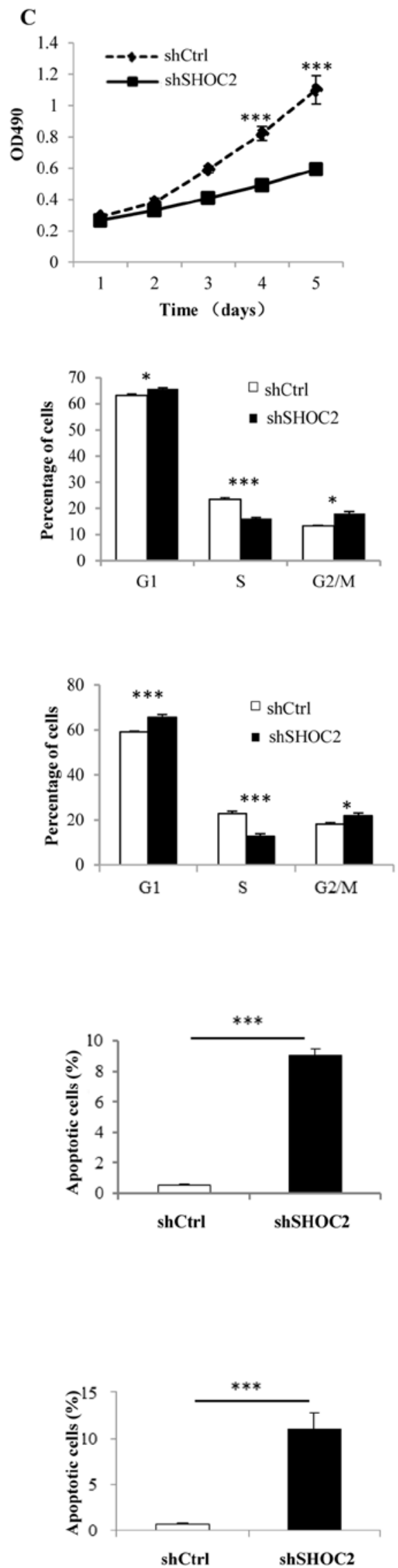

Figure 1. Effects of SHOC2 knockdown on proliferation, cell cycle progression and apoptosis of breast cancer cells. (A) Protein expression of SHOC2 after knockdown in MCF-7 and MDA-MB-231 breast cancer cells. MTT assay results of (B) MCF-7 and (C) MDA-MB-231 breast cancer cells. Cell cycle distribution of (D) MCF-7 and (E) MDA-MB-231 breast cancer cells, as determined by flow cytometry. Cell apoptosis analyzed using flow cytometry and Annexin V staining of (F) MCF-7 and (G) MDA-MB-231 breast cancer cells. For apoptosis analysis, the right quadrants were assessed. ${ }^{*} \mathrm{P}<0.05,{ }^{* * * *} \mathrm{P}<0.001 \mathrm{vs}$. shCtrl. Ctrl, control; OD, optical density; SHOC2, SHOC2 leucine rich repeat scaffold protein; sh, short hairpin RNA. 
A

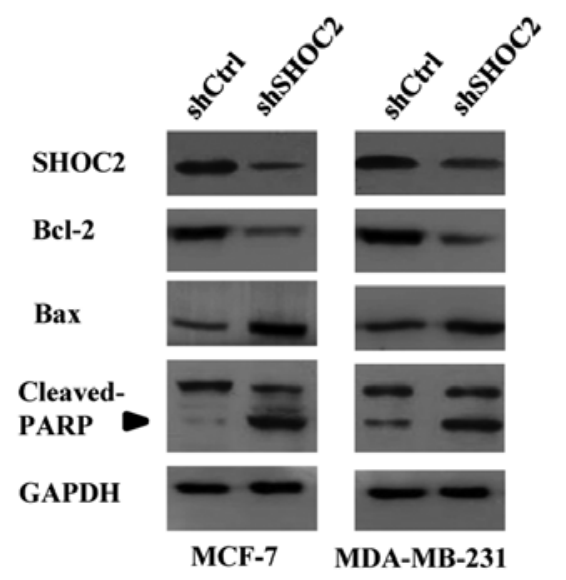

B

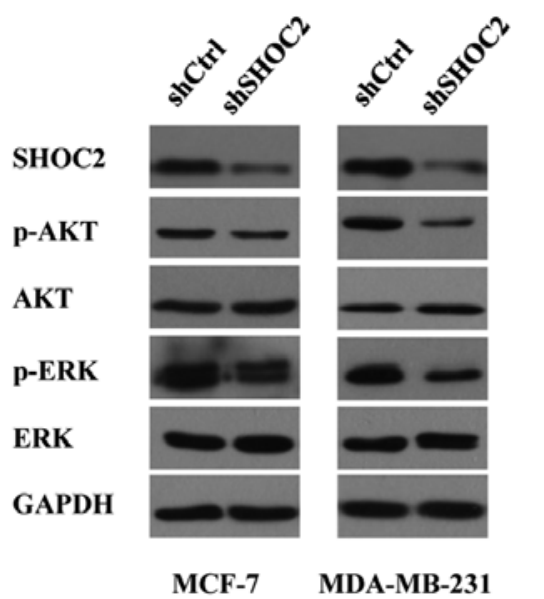

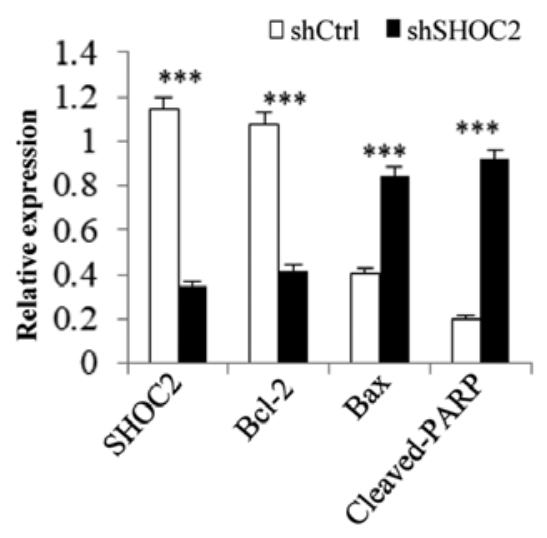

MCF-7

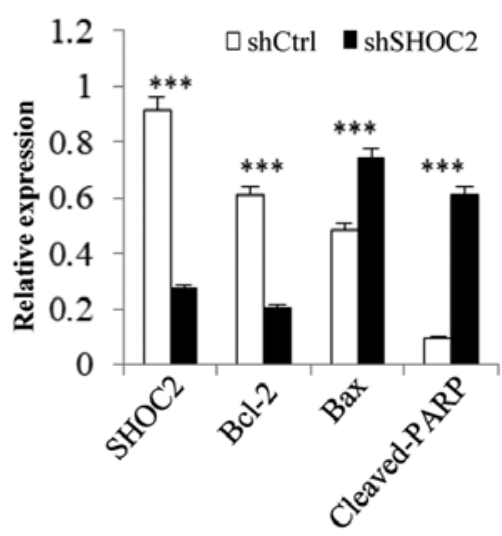

MDA-MB-231

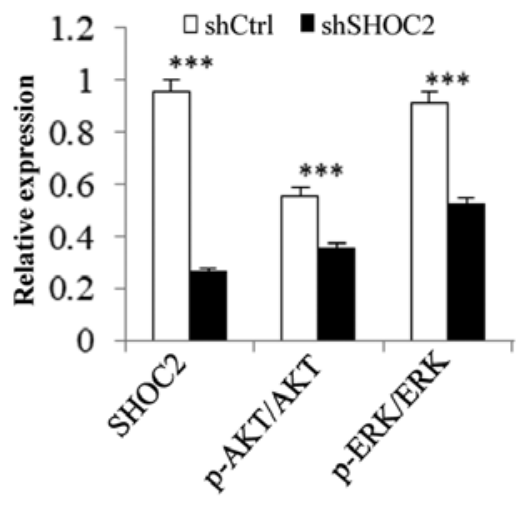

MCF-7

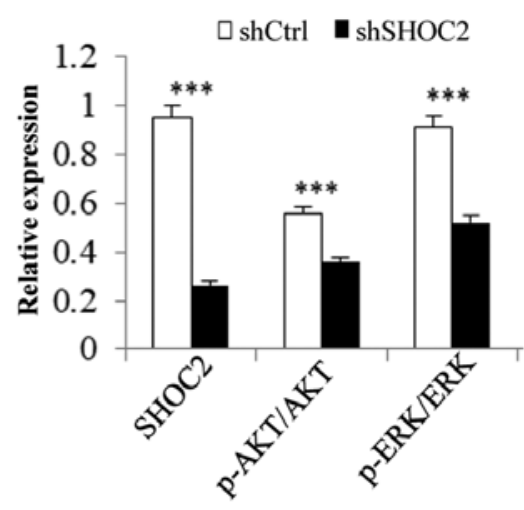

MDA-MB-231

Figure 2. Effects of SHOC2 knockdown on the expression of Ras signaling pathway and apoptosis-related proteins in breast cancer cells. (A) Protein expression of Bcl-2, Bax and cleaved-PARP following SHOC2 knockdown in MCF-7 and MDA-MB-231 breast cancer cells. (B) Protein expression of AKT, p-AKT, ERK and p-ERK following SHOC2 knockdown in MCF-7 and MDA-MB-231 breast cancer cells. ${ }^{* * *} \mathrm{P}<0.001$ vs. shCtrl. Ctrl, control; PARP, poly (ADP-ribose) polymerase; p, phosphorylated; SHOC2, SHOC2 leucine rich repeat scaffold protein; sh, short hairpin RNA.

The RAS-MAPK/PI3K pathway is inhibited by SHOC2 knockdown. Analysis of RAS-MAPK/PI3K pathway-associated protein expression revealed that after SHOC2 was knocked down in MCF-7 and MDA-MB-231 breast cancer cells, p-ERK and p-AKT expression was decreased. These findings indicated that the Ras-ERK and PI3K-AKT pathways were inhibited by SHOC 2 knockdown, as revealed by the decrease in positive signals for $\mathrm{p}$-ERK and $\mathrm{p}-\mathrm{AKT}$ ( $\mathrm{P}<0.001$; Fig. 2B).

SHOC2 is overexpressed in human breast cancer tissues. To explore the roles of SHOC2 in human breast cancer, SHOC2 mRNA was evaluated in fresh clinical samples by RT-qPCR. SHOC2 expression was significantly higher in human breast cancer tissues than in paired normal breast tissues $(\mathrm{P}<0.01$; Fig. 3A). In the IHC analysis, SHOC2 protein was predominantly found in the cytoplasm of tumor cells, and this demonstrated that 88 of $120(73.3 \%)$ breast cancer patients had high SHOC2 expression. Consistent with the RT-qPCR results, increased SHOC2 expression levels, compared with those in normal breast tissues, were detected in the tumor tissues $(\mathrm{P}<0.01$; Fig. 3B-F).

Association between SHOC2 expression and the clinicopathological features of breast cancer. The associations between SHOC2 expression and the clinicopathological features of breast cancer are summarized in Table I. The results showed that significant associations were observed between high SHOC2 expression and high histopathological grades $(\mathrm{P}<0.01)$. Similarly, regarding tumor size, SHOC2 expression scores were higher in T2-T3 $(>2 \mathrm{~cm})$ tumors than in $\mathrm{T} 1(\leq 2 \mathrm{~cm})$ tumors $(\mathrm{P}=0.023)$. In addition, SHOC2 expression 
A

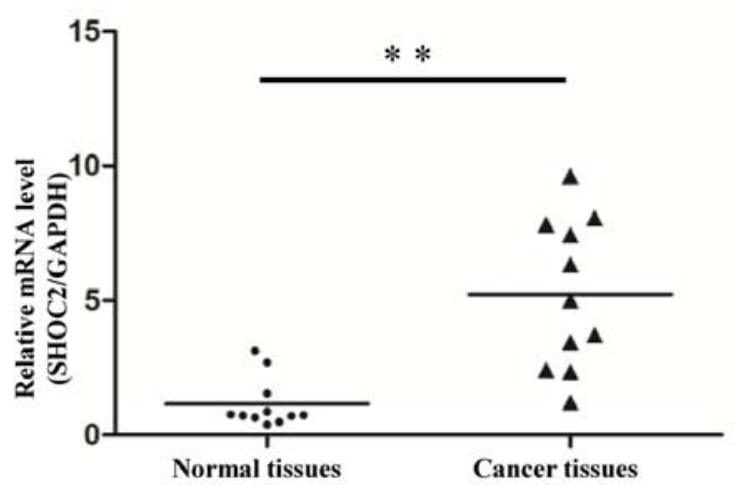

C

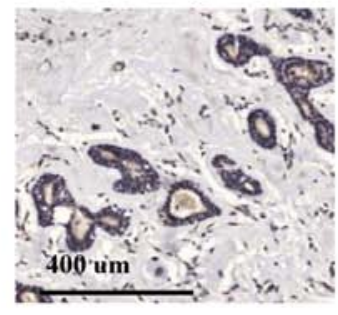

D

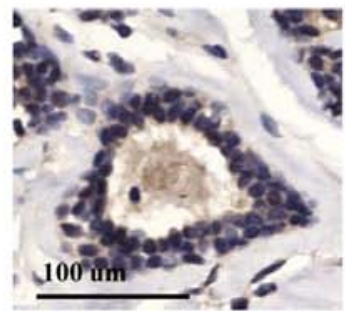

E

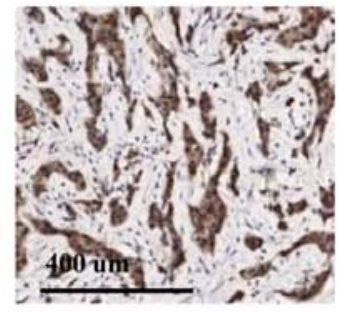

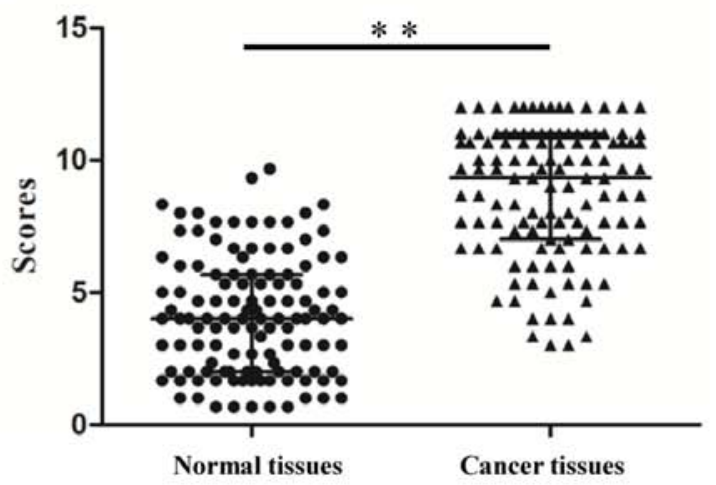

G

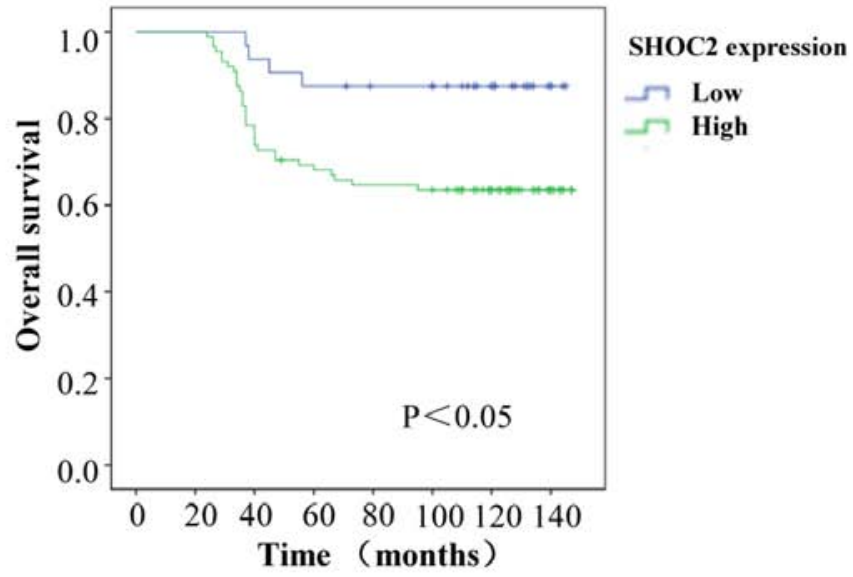

H

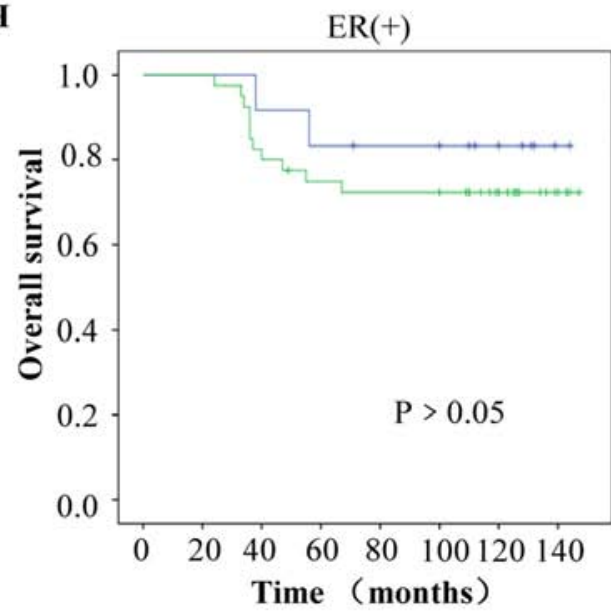

I

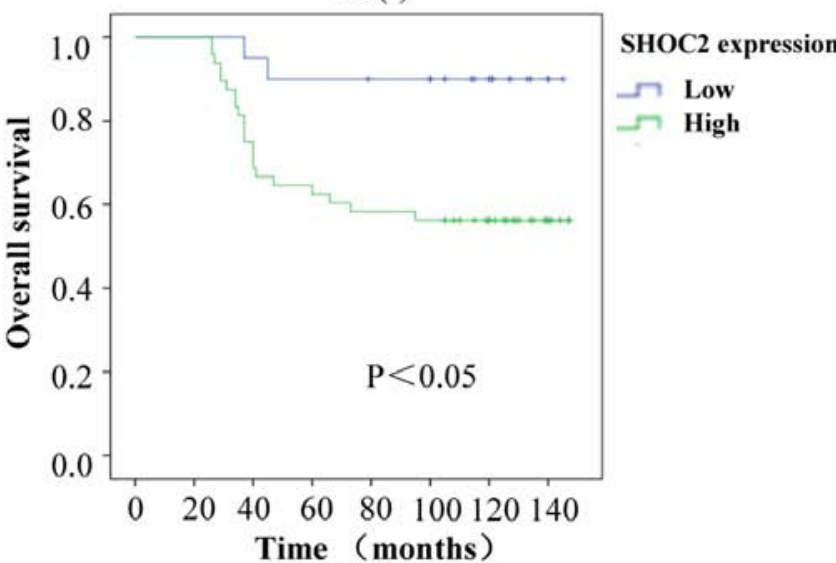

Figure 3. Clinical association and prognostic value of SHOC2 expression for breast cancer. (A) Relative mRNA expression levels of SHOC2 in fresh clinical samples. (B) IHC staining scores for the normal breast and invasive breast cancer tissues. (C and D) IHC staining shows that SHOC2 expression was low in normal breast tissues; (C) magnification, x50 and (D) magnification, x200. (E and F) IHC staining shows that SHOC2 was upregulated in invasive breast cancer tissues; (E) magnification, x50 and (F) magnification, x200. (G) Kaplan-Meier plot showing a significant association between SHOC2 expression and OS for patients with breast cancer. (H) In the ER (+) subgroups, OS rates were not significantly affected by SHOC2 expression. (I) In the ER (-) subgroups, OS rates were significantly affected by SHOC2 expression. ${ }^{* *} \mathrm{P}<0.01$. ER, estrogen receptor; IHC, immunohistochemistry; OS, overall survival; SHOC2, SHOC2 leucine rich repeat scaffold protein.

was associated with estrogen receptor (ER) status and was higher in patients with ER-negative breast cancer than in patients with ER-positive breast cancer $(\mathrm{P}=0.028)$. However, the associations between SHOC2 expression and patient age, progesterone receptor, HER2 or lymph node status were not statistically significant. 
Table I. Association between SHOC2 expression and clinicopathological features of patients with breast cancer.

\begin{tabular}{|c|c|c|c|c|}
\hline \multirow[b]{2}{*}{ Characteristics } & \multirow{2}{*}{$\frac{\text { Total no. }}{120}$} & \multicolumn{2}{|c|}{$\begin{array}{c}\text { SHOC2 } \\
\text { expression }\end{array}$} & \multirow[b]{2}{*}{ P-value } \\
\hline & & Low & High & \\
\hline Age & & & & 0.366 \\
\hline$\leq 40$ years & 35 & 7 & 28 & \\
\hline$>40$ years & 85 & 25 & 60 & \\
\hline Tumor size & & & & 0.023 \\
\hline$\leq 2 \mathrm{~cm}$ & 57 & 21 & 36 & \\
\hline $2-5 \mathrm{~cm}$ & 63 & 11 & 52 & \\
\hline Histopathological grade & & & & $<0.001$ \\
\hline I & 34 & 18 & 16 & \\
\hline II & 53 & 9 & 44 & \\
\hline III & 33 & 5 & 28 & \\
\hline ER & & & & 0.028 \\
\hline+ & 40 & 16 & 24 & \\
\hline- & 80 & 16 & 64 & \\
\hline PR & & & & 0.533 \\
\hline+ & 68 & 20 & 48 & \\
\hline- & 52 & 12 & 40 & \\
\hline HER 2 & & & & 0.536 \\
\hline+ & 76 & 20 & 56 & \\
\hline- & 44 & 12 & 32 & \\
\hline Lymph node status & & & & 0.371 \\
\hline+ & 86 & 21 & 65 & \\
\hline- & 34 & 11 & 23 & \\
\hline
\end{tabular}

ER, estrogen receptor; HER2, human epidermal growth factor receptor 2; PR, progesterone receptor; SHOC2, SHOC2 leucine rich repeat scaffold protein.

SHOC2 expression is an independent prognostic factor for the survival of patients with breast cancer. The Kaplan-Meier method was applied to explore the significance of SHOC2 expression for the OS rates of the enrolled patients. The results revealed that patients with low SHOC2 expression had improved OS than those with high SHOC2 expression (Fig. 3G). The prognostic value of SHOC2 expression was analyzed in different subgroups of patients. For the patients with different SHOC2 expression statuses, differences in prognosis were more notable in the ER-negative subgroup than in the ER-positive subgroup. The OS was significantly lower for patients with high SHOC2 expression than for patients with low expression $(\mathrm{P}<0.05$; Fig. $3 \mathrm{H}$ and $\mathrm{I})$; this result implied that the expression of SHOC2 may be more important for ER-negative patients. The univariate analysis revealed that tumor size $(\mathrm{P}=0.023)$, ER status $(\mathrm{P}=0.015)$, lymph node status $(\mathrm{P}<0.001)$ and $\mathrm{SHOC} 2$ expression $(\mathrm{P}=0.020)$ were all related to OS (Table II). These data suggested that SHOC2 might have prognostic value for patients with breast cancer. Subsequently, a multivariate analysis was performed using the Cox proportional hazards model and demonstrated that high SHOC2 expression $(\mathrm{P}<0.001)$, lymph node status $(\mathrm{P}<0.001)$ and negative ER expression status $(\mathrm{P}=0.010)$ were independent prognostic factors for survival (Table II).

\section{Discussion}

It is commonly known that high frequency mutations of Ras serve a crucial role in the development and progression of various types of cancer, including $90 \%$ of pancreatic cancers. In contrast, Ras mutations are rare in breast cancer ( 5\%) (23). Despite the low frequency of mutations, considerable research has shown that abnormal activation of the Ras pathway promotes breast cancer development, and Ras is activated in numerous breast cancer cell lines, including MCF-7 and MDA-MB-231 (24). Studies have demonstrated that even in the presence of Ras mutations, interfering with SHOC2 expression can still affect the proliferation and apoptosis of cancer cells $(8,25)$. Therefore, as a key scaffold protein in activation of the Ras pathway, SHOC2 may have an important role in breast cancer. In the present study, shRNA was generated to assess the potential role of SHOC2 in cancer cell proliferation. The results showed that SHOC2 knockdown could significantly decrease cell numbers and inhibit breast cancer cell proliferation. Furthermore, SHOC2 knockdown resulted in apoptosis and cell cycle arrest in breast cancer cells. To explore the mechanism underlying the effects of SHOC2 on breast cancer cells, the Ras pathway was examined, for which SHOC2 is required (26). The results showed that both the PI3K/AKT and MAPK/ERK pathways were inhibited by SHOC2 knockdown, which supported previous research into colorectal carcinoma (12).

In the present study, clinical data were also analyzed, the results revealed that $\mathrm{SHOC} 2$ protein was frequently expressed in breast tumors and associated with clinicopathological variables important for disease outcome. Additionally, to the best of our knowledge, this study was the first to reveal that the difference in SHOC2 expression between breast cancer and normal breast tissue was significant $(\mathrm{P}<0.01)$, suggesting that SHOC2 may play an important role in breast cancer progression. To assess the clinical significance of SHOC2 protein expression in breast cancer, the association between a number of clinicopathological characteristics and SHOC2 protein expression status was assessed in the breast tumor samples. It was observed that $\mathrm{SHOC} 2$ expression was more abundant in $\mathrm{T} 2-\mathrm{T} 3$ tumors than in $\mathrm{T} 1$ tumors $(\mathrm{P}=0.023)$. Similarly, patients with higher SHOC2 expression had higher histological grade tumors than patients with low SHOC2 protein expression $(\mathrm{P}<0.01)$. Therefore, SHOC2 upregulation is associated with worse clinical features.

The effect of SHOC2 expression on patient survival was estimated by Kaplan-Meier analysis. Patients with breast cancer and high SHOC2 protein expression had a significantly worse prognosis than patients with low SHOC2 protein expression $(\mathrm{P}<0.05)$. Notably, in the subgroup analysis, it was revealed that patients in the ER-negative subgroup with low SHOC2 expression had an improved prognosis than those with high SHOC2 expression. Therefore, SHOC2 may serve a more important role in patients with ER-negative breast cancer than in patients with ER-positive breast cancer $(\mathrm{P}<0.05)$. In addition, the results demonstrated that high SHOC2 expression, 
Table II. Univariate and multivariate survival analyses of various factors in patients with breast cancer.

\begin{tabular}{|c|c|c|c|c|c|c|}
\hline \multirow[b]{2}{*}{ Characteristics } & \multicolumn{3}{|c|}{ Univariate } & \multicolumn{3}{|c|}{ Multivariate } \\
\hline & $\mathrm{HR}$ & $95 \% \mathrm{CI}$ & P-value & $\mathrm{HR}$ & $95 \% \mathrm{CI}$ & P-value \\
\hline \multicolumn{7}{|l|}{ Age } \\
\hline$\leq 40$ years & 1 & & & & & \\
\hline$>40$ years & 0.765 & $0.387-1.510$ & 0.439 & & & \\
\hline \multicolumn{7}{|l|}{ Tumor size } \\
\hline$\leq 2 \mathrm{~cm}$ & 1 & & & 1 & & \\
\hline$>2 \mathrm{~cm}$ & 2.280 & $1.121-4.635$ & 0.023 & 1.077 & $0.507-2.288$ & 0.847 \\
\hline \multicolumn{7}{|c|}{ Histopathological grade } \\
\hline $\mathrm{I}$ & 1 & & & & & \\
\hline II & 1.019 & $0.462-2.244$ & 0.964 & & & \\
\hline III & 1.102 & $0.726-1.674$ & 0.648 & & & \\
\hline \multicolumn{7}{|l|}{ ER } \\
\hline- & 1 & & & 1 & & \\
\hline+ & 0.444 & $0.231-0.854$ & 0.015 & 0.415 & $0.212-0.812$ & 0.010 \\
\hline \multicolumn{7}{|l|}{ PR } \\
\hline- & 1 & & & & & \\
\hline+ & 0.703 & $0.356-1.387$ & 0.309 & & & \\
\hline \multicolumn{7}{|l|}{ HER2 } \\
\hline- & 1 & & & & & \\
\hline+ & 1.087 & $0.560-2.108$ & 0.805 & & & \\
\hline \multicolumn{7}{|c|}{ Lymph node status } \\
\hline- & 1 & & & 1 & & \\
\hline+ & 4.984 & $2.561-9.700$ & $<0.01$ & 5.262 & $2.590-10.691$ & $<0.001$ \\
\hline \multicolumn{7}{|c|}{ SHOC2 expression } \\
\hline Low & & & & 1 & & \\
\hline High & 3.428 & $1.212-9.697$ & 0.020 & 5.440 & $1.865-15.863$ & $<0.001$ \\
\hline
\end{tabular}

ER, estrogen receptor; HER2, human epidermal growth factor receptor 2; PR, progesterone receptor; SHOC2, SHOC2 leucine rich repeat scaffold protein.

large tumor size, ER-negative status and positive lymph node status were independent prognostic factors for this disease. These data suggested that SHOC2 upregulation was related to poor prognosis and could act as a therapeutic target in breast cancer. As shown in this study, the clinical effects of SHOC2 on patients with ER-negative breast cancer were more significant. Previous studies have demonstrated that the Ras pathway, in which SHOC2 has a major role, is more active in ER-negative breast tumors $(27,28)$.

However, there were several limitations of the present study. Firstly, only one shRNA target sequence was used to knockdown SHOC2; it would have been useful to use more. Although, to avoid the off-target effects, two cell lines were used to verify its reliability. Secondly, even though the present study included 120 patients, they were recruited from a single center so this study suffered from selection bias. Additionally, the rate of disease-free survival (DFS; the time to recurrence or distant metastasis) of the patients was recorded during initial follow-ups, but these patients all passed away in further follow-ups. This meant that the effect of SHOC2 expression on
DFS and OS are similar in this research so only the OS results were shown, which is another limitation to the present study. More clinical and experimental studies are required to define the genetic mechanisms in order to further understand the role of SHOC2 in normal mammary gland and breast cancer tissues.

In conclusion, it was found that SHOC2 knockdown has a significant effect on breast cancer cell proliferation, as well as the induction of cell cycle arrest and apoptosis. Additionally, SHOC2 was overexpressed in breast cancer and was associated with the OS of patients with breast cancer. The biological function of SHOC2 was examined in this study, and these results suggested that $\mathrm{SHOC} 2$ could be a therapeutic target for breast cancer.

\section{Acknowledgements}

The authors would like to thank Dr Qianqian Zhao and Dr Haiyun Song of the Pathology Department, Qilu Hospital (Qingdao) of Shandong University for their help in the IHC experiment. 


\section{Funding}

This study was funded by The NSFC (grant no. 81572587) and The Shandong Natural Science Fund (grant no.ZR2018PH029).

\section{Availability of data and materials}

The datasets used and/or analyzed during the current study are available from the corresponding author on reasonable request.

\section{Authors' contributions}

HG designed the experiments. WG, KD and YL performed the experiments. WG and QP drafted the manuscript, and QP performed statistical analysis and figure illustration. YL provided experimental technical guidance. All authors read and approved the final manuscript.

\section{Ethics approval and consent to participate}

This study has been approved by the Research Ethics Committee of Qilu Hospital (Qingdao) of Shandong University (policy no. KYLL-2016038). All procedures performed in the studies involving human participants were in accordance with the ethical standards of the Research Ethics Committee of Qilu Hospital (Qingdao) of Shandong University and with The Declaration of Helsinki (1964) and its later amendments or comparable ethical standards. Informed consent was obtained from all individual participants included in the study.

\section{Patient consent for publication}

Not applicable.

\section{Competing interests}

The authors declare that they have no competing interests.

\section{References}

1. Ban KA and Godellas CV: Epidemiology of breast cancer. Surg Oncol Clin N Am 23: 409-422, 2014.

2. Lee EY and Muller WJ: Oncogenes and tumor suppressor genes. Cold Spring Harb Perspect Biol 2: a003236, 2010.

3. Downward J: Targeting RAS signalling pathways in cancer therapy. Nat Rev Cancer 3: 11-22, 2003.

4. Sieburth DS, Sun Q and Han M: SUR-8, a conserved Ras-binding protein with leucine-rich repeats, positively regulates Ras-mediated signaling in C. elegans. Cell 94: 119-130, 1998.

5. Jang ER, Jang H, Shi P, Popa G, Jeoung M and Galperin E: Spatial control of Shoc2-scaffold-mediated ERK1/2 signaling requires remodeling activity of the ATPase PSMC5. J Cell Sci 128: 4428-4441, 2015.

6. Matsunaga-Udagawa R, Fujita Y, Yoshiki S, Terai K, Kamioka Y, Kiyokawa E, Yugi K, Aoki K and Matsuda M: The scaffold protein Shoc2/SUR-8 accelerates the interaction of Ras and Raf. J Biol Chem 285: 7818-7826, 2010.

7. Li W, Han M and Guan KL: The leucine-rich repeat protein SUR-8 enhances MAP kinase activation and forms a complex with Ras and Raf. Genes Dev 14: 895-900, 2000.

8. Rodriguez-Viciana P, Oses-Prieto J, Burlingame A, Fried M and McCormick F: A phosphatase holoenzyme comprised of Shoc2/Sur8 and the catalytic subunit of PP1 functions as an M-Ras effector to modulate Raf activity. Mol Cell 22: 217-230, 2006.

9. Shaw AS and Filbert EL: Scaffold proteins and immune-cell signalling. Nat Rev Immunol 9: 47-56, 2009.
10. Rouchka EC, Jeoung M, Jang ER, Liu J, Wang C, Li X and Galperin E: Data set for transcriptional response to depletion of the Shoc2 scaffolding protein. Data Brief 7: 770-778, 2016.

11. Young LC, Hartig N, Munoz-Alegre M, Oses-Prieto JA, Durdu S, Bender S, Vijayakumar V, Vietri Rudan M, Gewinner C, Henderson S, et al: An MRAS, SHOC2, and SCRIB complex coordinates ERK pathway activation with polarity and tumorigenic growth. Mol Cell 52: 679-692, 2013.

12. Lee YM, Kaduwal S, Lee KH, Park JC, Jeong WJ and Choi KY: Sur8 mediates tumorigenesis and metastasis in colorectal cancer. Exp Mol Med 48: e249, 2016.

13. Kaduwal S, Jeong WJ, Park JC, Lee KH, Lee YM, Jeon SH, Lim YB, Min do S and Choi KY: Sur8/Shoc2 promotes cell motility and metastasis through activation of Ras-PI3K signaling. Oncotarget 6: 33091-33105, 2015.

14. Kaplan FM, Kugel CH III, Dadpey N, Shao Y, Abel EV and Aplin AE: SHOC2 and CRAF mediate ERK1/2 reactivation in mutant NRAS-mediated resistance to RAF inhibitor. J Biol Chem 287: 41797-41807, 2012.

15. Traini S, Piccolo E, Tinari N, Rossi C, La Sorda R, Spinella F, Bagnato A, Lattanzio R, D'Egidio M, Di Risio A, et al: Inhibition of tumor growth and angiogenesis by SP-2, an anti-lectin, galactoside-binding soluble 3 binding protein (LGALS3BP) antibody. Mol Cancer Ther 13: 916-925, 2014.

16. Jeoung M, Jang ER, Liu J, Wang C, Rouchka EC, Li X and Galperin E: Shoc2-tranduced ERK1/2 motility signals--Novel insights from functional genomics. Cell Signal 28: 448-459, 2016.

17. Aran D, Camarda R, Odegaard J, Paik H, Oskotsky B, Krings G, Goga A, Sirota M and Butte AJ: Comprehensive analysis of normal adjacent to tumor transcriptomes. Nat Commun 8: 1077, 2017.

18. Li C, Cao L, Xu C, Liu F, Xiang G, Liu X, Jiao J and Niu Y: The immunohistochemical expression and potential prognostic value of HDAC6 and AR in invasive breast cancer. Hum Pathol 75: 16-25, 2018.

19. Ye W, Chen C, Gao Y, Zheng ZS, Xu Y, Yun M, Weng HW, Xie D, Ye $S$ and Zhang JX: Overexpression of SLC34A2 is an independent prognostic indicator in bladder cancer and its depletion suppresses tumor growth via decreasing c-Myc expression and transcriptional activity. Cell Death Dis 8: e2581, 2017.

20. Shen Q, Yao Q, Sun J, Feng L, Lu H, Ma Y, Liu L, Wang F, $\mathrm{Li} \mathrm{J}$, Yue Y, et al: Downregulation of histone deacetylase 1 by microRNA-520h contributes to the chemotherapeutic effect of doxorubicin. FEBS Lett 588: 184-191, 2014.

21. Lin X, Yu Y, Zhao H, Zhang Y, Manela J and Tonetti DA: Overexpression of PKCalpha is required to impart estradiol inhibition and tamoxifen-resistance in a T47D human breast cancer tumor model. Carcinogenesis 27: 1538-1546, 2006.

22. Livak KJ and Schmittgen TD: Analysis of relative gene expression data using real-time quantitative PCR and the 2(-Delta Delta C(T)) method. Methods 25: 402-408, 2001.

23. Clark GJ and Der CJ: Aberrant function of the Ras signal transduction pathway in human breast cancer. Breast Cancer Res Treat 35: 133-144, 1995.

24. Eckert LB, Repasky GA, Ulku AS, McFall A, Zhou H, Sartor CI and Der CJ: Involvement of Ras activation in human breast cancer cell signaling, invasion, and anoikis. Cancer Res 64: 4585-4592, 2004.

25. Jones GG, Del Rio IB, Sari S, Sekerim A, Young LC, Hartig N, Areso Zubiaur I, El-Bahrawy MA, Hynds RE, Lei W, et al: SHOC2 phosphatase-dependent RAF dimerization mediates resistance to MEK inhibition in RAS-mutant cancers. Nat Commun 10: 2532, 2019.

26. Jang ER and Galperin E: The function of Shoc2: A scaffold and beyond. Commun Integr Biol 9: e1188241, 2016.

27. Loboda A, Nebozhyn M, Klinghoffer R, Frazier J, Chastain M, Arthur W, Roberts B, Zhang T, Chenard M, Haines B, et al: A gene expression signature of RAS pathway dependence predicts response to PI3K and RAS pathway inhibitors and expands the population of RAS pathway activated tumors. BMC Med Genomics 3: 26, 2010.

28. Li W, Liang RR, Zhou C, Wu MY, Lian L, Yuan GF, Wang MY, Xie X, Shou LM, Gong FR, et al: The association between expressions of Ras and CD68 in the angiogenesis of breast cancers. Cancer Cell Int 15: 17, 2015.

This work is licensed under a Creative Commons Attribution-NonCommercial-NoDerivatives 4.0 International (CC BY-NC-ND 4.0) License. 